\title{
miR-424 targets AKT3 and PSATI and has a tumor-suppressive role in human colorectal cancer
}

This article was published in the following Dove Press journal: Cancer Management and Research

\author{
Yifeng Fang' \\ Xiao Liang' \\ Junfen $X u^{2}$ \\ Xiujun Cai ${ }^{\prime}$ \\ 'Department of General Surgery, \\ Sir Run Run Shaw Hospital, Zhejiang \\ University School of Medicine, \\ Hangzhou, China; ${ }^{2}$ Department of \\ Gynecologic Oncology, Women's \\ Hospital, Zhejiang University School \\ of Medicine, Hangzhou, China
}

Background: Recent advances in cancer biology have uncovered critical roles for microRNAs in regulating tumor responses. This study is to elucidate the role of miR-424 in colorectal cancer development.

Materials and methods: miR-424 expression was analyzed by qRT-PCR. The role of miR424 was studied in cell lines and animal models. The downstream targets of miR-424 were determined by microarray analysis.

Results: We found that miR-424 expression was downregulated in human colorectal cancer cell lines and patient biopsies. We demonstrated that miR-424 functioned as a tumor suppressor by suppressing colorectal cancer growth in vitro and in vivo and enhancing apoptosis. Using microarray screening, we subsequently presented evidence that miR-424 directly targeted the 3' untranslated regions of the AKT serine/threonine kinase 3 (AKT3) and phosphoserine aminotransferase 1 (PSAT1) mRNAs via luciferase assay. Furthermore, AKT3 or PSAT1 silencing partially recapitulated the effects of miR-424.

Conclusion: This newly identified miR-424/AKT3-SAT1 axis may represent a novel therapeutic strategy for future treatment of colorectal cancer.

Keywords: miR-424, AKT3, PSAT1, colorectal cancer

\section{Introduction}

Colorectal cancer $(\mathrm{CRC})$ represents the third most common malignancy worldwide accounting for estimated 1.4 million cases and 693,900 deaths annually. ${ }^{1}$ In spite of the decreasing incidence and mortality, CRC remains a major public health problem. Multiple risk factors have been reported to contribute to this cancer, such as unhealthy diet, obesity, and smoking. ${ }^{2}$ Even though the 5-year survival rate of CRC patients at an early stage is about $90 \%$, only $39 \%$ of cancers are diagnosed at this stage. The 5-year survival rate drops to $12.5 \%$ when the disease has spread to distant organs. ${ }^{3}$ Therefore, it is important to uncover the molecular mechanisms underlying CRC, to identify therapeutic targets to improve treatment.

MicroRNAs (miRNAs), a class of 21-24-nucleotide (nt) noncoding RNA, regulate gene expression by binding to the $3^{\prime}$ untranslated region (UTR) of mRNAs containing complementary sequences, thereby resulting in translational repression and degradation of the target mRNAs. ${ }^{4,5}$ Well over half of the human transcriptome is predicted to be under the regulation of miRNAs, it is not surprising that aberrant miRNA expression has a functional role in many human diseases, including cancer. ${ }^{4,6-8}$ The initial implicated miRNAs in CRC were miR-145 and miR-143, which were reduced 
in CRC. Then, many other miRNAs have been identified as oncogenic or tumor suppressive miRNAs in the initiation and progression of CRC.

In this study, we characterized miR-424 that is frequently downregulated in CRC. This miRNA is capable of inhibiting proliferation and inducing apoptosis of CRC cells. We analyzed the gene expression profiles following miR-424 reintroduction in HCT116 cells through microarrays. MiR-424 and its potential target relationships were further constructed according to the microarray results and bioinformatics predictions. We ultimately identified the AKT serine/threonine kinase 3 (AKT3) and phosphoserine aminotransferase 1 (PSAT1) as the direct targets for miR-424, and they were involved in miR-424-mediated apoptosis. Our study reveals key roles of miR-424 and its targets AKT3 and PSAT1 in CRC, some with immediate implications for therapeutic exploration.

\section{Materials and methods}

\section{Tissue specimens}

Human CRC samples and paired normal mucosa were collected from patients who underwent surgical resection of CRC at the Department of General Surgery in Sir Run Run Shaw Hospital, Zhejiang University, China. This study was approved by Human Research Ethics Committee of the hospital and conducted in accordance with the Declaration of Helsinki. Written informed consent was obtained from each patient. Both tumor and normal tissues were histologically confirmed. All tissue samples were selected immediately after surgical resection, and stored at $-80^{\circ} \mathrm{C}$.

\section{Cell culture and transfection}

Human colon cancer cell lines HCT116 and RKO were bought from the American Type Culture Collection (ATCC, Manassas, VA, USA). HCT116 was maintained as monolayers in McCOY'S 5A (Corning Incorporated, Corning, NY, USA) and RKO in RPMI 1640 (Corning Incorporated), respectively.

MiR-424 mimic and nonspecific negative control miRNA (miR-NC) were purchased from Dharmacon, Lafayette, CO, USA. Specific AKT3 siRNA, PSAT1 siRNA, and negative control siRNA (NC) were synthesized by GenePharma, Shanghai, China.

MiRNA mimic or siRNA was reversely transfected using DharmaFECT 1 transfection reagent (Dharmacon). A final concentration of both $50 \mathrm{nM}$ siRNA and miRNA mimic was used for each transfection. Cotransfection of the miRNA and plasmid DNA was conducted using DharmaFECT Duo transfection reagent (Dharmacon). Transfections were performed according to the manufacturer's protocol.

\section{Quantitative real-time PCR}

RNA extraction and real-time quantitative reverse transcriptasePCR (RT-qPCR) was performed as described previously. ${ }^{9}$ Briefly, RNA was extracted from primary colorectal tissues or cultured cells using Trizol reagent (Thermo Fisher Scientific, Waltham, MA, USA). For miR-424, stem-loop RT-PCR was performed. For mRNA analysis, cDNA was generated from $5 \mu \mathrm{g}$ total RNA per sample using the PrimeScript RT reagent Kit (TaKaRa). qPCR was performed using SYBR Premix Ex Taq (TaKaRa). PCR-specific amplification was conducted on an Applied Biosystems ViiA 7 Dx real-time PCR machine (Thermo Fisher Scientific). The relative expression of miR-424 or genes was calculated by the $2^{-(\Delta \Delta C T)}$ method and normalized to U6 or glyceraldehyde 3-phosphate dehydrogenase (GAPDH).

\section{Cell proliferation assay}

Cells were seeded in 96-well plates with 4,000 cells per well, transfected with miRNA mimic or siRNA, respectively. The cells were then cultured for 1, 2, 3, or 4 days. The cell proliferation was tested by CCK-8 kit (Dojindo, Kumamoto, Japan). Absorbance was determined at $450 \mathrm{~nm}$ on a microplate reader (Thermo Fisher Scientific). The proliferation curve was generated based on the absorbance and time. Transfections were performed in triplicate and repeated in at least three independent experiments.

\section{Animal experiments}

Four-week-old BALB/c nude mice were obtained from Shanghai Laboratory Animal Center. All animal studies were approved by the Animal Care Committee of Zhejiang University. Animal experiments complied with the ARRIVE guidelines and followed the National Institutes of Health Guide for the Care and Use of Laboratory Animals. After 5 days of acclimatization, a total of $5 \times 10^{6}$ HCT116 cells stably transfected with either miR-424 or miR-NC were injected subcutaneously in the left-side axilla of each mouse ( $n=3 /$ each group). The tumor volume was calculated using the formula (lengh $\times$ depth $\times$ width) $\times \pi / 6$. The mice were killed on the 34th day after transplantation, and tumors were removed, weighed, and photographed.

\section{Annexin- $\mathrm{V}$ binding assay}

Apoptosis was analyzed using a FACS Annexin V and PI assay kit (Biouniquer, China). Cells were transfected with miRNA mimics or siRNAs and incubated for 72 hours. Samples were analyzed on a FACScan flow cytometer (FACSCalibur ${ }^{\mathrm{TM}}$; BD, Franklin Lakes, NJ, USA). The experiments were performed in duplicate and repeated at least three times. 


\section{Microarray-based gene expression analysis}

The Agilent Human Gene Expression (Design ID:039494) was used in this experiment. The arrays were performed by the Agilent Scanner G2505C (Agilent Technologies, Santa Clara, CA, USA). Feature Extraction software (version10.7.1.1, Agilent Technologies) and Genespring were used to get raw data and finish the basic analysis, respectively. The probes in that at least one out of all samples has flags in Detected were chosen. Differentially expressed genes were then determined through $P$-value (calculated with Student's $t$-test) as well as fold change. Up- and downregulated genes were identified by a $P$-value $<0.05$ and an absolute fold change $\geq 2.0$.

\section{Ingenuity pathway analysis (IPA)}

The differentially expressed genes were submitted to IPA (Ingenuity Systems, Qiagen, Germany) (www.ingenuity. com). Canonical pathway analysis, molecular and cell function enrichment analysis, and network analysis were done. For IPA, 499 differentially expressed genes in the comparison between miR-424 and miR-NC groups were first uploaded for the purpose of generating gene symbol, fold change, and $P$-value data. Finally, the functional annotation identifies the biologic roles that are the most significant to the data set.

\section{Western blotting}

Western blotting was performed as described elsewhere ${ }^{10,11}$ with the following antibodies: anti-AKT3 (Sigma-Aldrich Co., St Louis, MO, USA), anti-PSAT1 (Sigma), and antiGAPDH (Santa Cruz Biotechnology, Inc., Dallas, TX, USA). Chemiluminescence signals were visualized with SuperSignal West Pico Chemiluminescent Substrate (Thermo Fisher Scientific) in a LAS-4000 image analyzer (Fuji Photo Film, Japan). The expression levels of these proteins were evaluated by Quantity One software.

\section{Luciferase reporter assays}

AKT3-3'UTR, AKT3-3'UTR-mut, PSAT1-3'UTR, and PSAT1-3'UTR-mut were constructed into pmirGLO dualluciferase miRNA target expression vector (Promega, Corporation, Fitchburg, WI, USA). Plasmids and miR-424 mimic or miR-NC were cotransfected into $293 \mathrm{~T}$ cells $\left(1 \times 10^{4}\right)$. The luciferase activity was examined using the dual-Glo Luciferase Reporter Assay System (Promega) by measuring the intensity of chemiluminescence in a luminometer (Thermo Fisher Scientific). Renilla luciferase activity was used as an internal control. Transfections were performed in triplicate and repeated in at least three independent experiments.

\section{Statistical analysis}

GraphPad Prism software 5.0 was used for statistical analysis. Statistical comparisons between each group were conducted with Student's $t$-test. $P$-value $<0.05$ indicates statistical significance.

\section{Results \\ miR-424 expression is aberrantly downregulated in CRC tissues}

To investigate the expression level of miR-424 in human CRC, we evaluated its expression in 53 matched pairs of CRC and corresponding normal colorectal tissues by RTqPCR. We found that the expression level of miR-424 was clearly reduced in CRC tissues compared to adjacent normal colorectal tissues (Figure 1A). Clinicopathologic analyses of the above 53 CRC patients showed that the miR-424 expression was negatively correlated with advanced clinical stage and lymph node metastasis (Table 1). However, the specific function of miR-424 in human CRC remained elusive.

\section{MiR-424 inhibits CRC cell growth in vitro and in vivo}

The similar expression pattern of miR-424 was also found in multiple CRC cell lines, as compared with normal colorectal tissues, further suggesting misregulation of miR-424 as a frequent event in human CRC (Figure 1B). The reduced expression of miR-424 in CRC tissues and cell lines prompted us to investigate the role of miR-424 in tumorigenesis. MiR424 was first ectopically expressed via miR-424 mimics in HCT116 and RKO cell lines, both of which have low levels of miR-424. Cell proliferation assays showed that miR-424 overexpression significantly suppressed CRC cell proliferation in both HCT116 and RKO cells (Figure 1C, D).

To confirm whether miR-424 inhibits CRC cell growth in vivo, HCT116 cell line that was stably transfected with miR-424 or control vector were implanted subcutaneously into nude mice. The tumors in the nude mice inoculated with HCT116/miR-424 cells grew much more slowly than those in the nude mice inoculated with HCT116/vector cells (Figure 1E). Thirty-four days after injection, the weights of the tumors derived from the HCT116/miR-424 cells were significantly lower than the weights of those from the control group (Figure 1F). Overall, our results indicate that miR-424 inhibits CRC cell growth both in vitro and in vivo. 
A

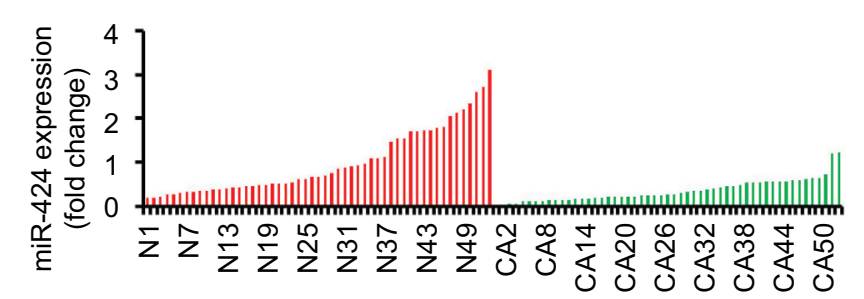

B

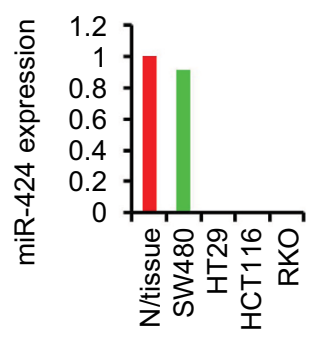

C

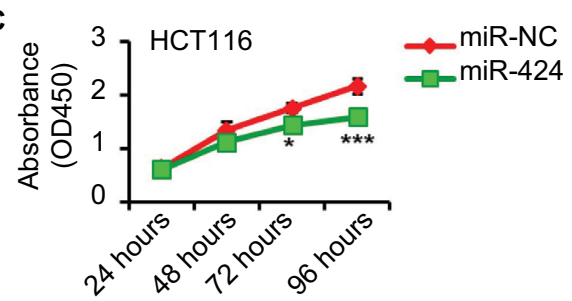

D

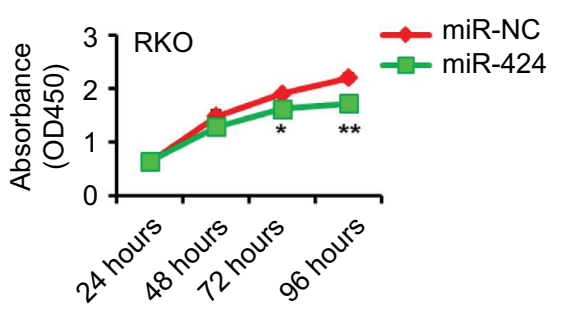

E

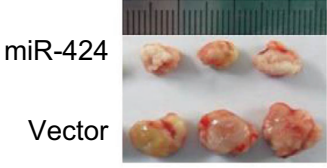

$\mathbf{F}$

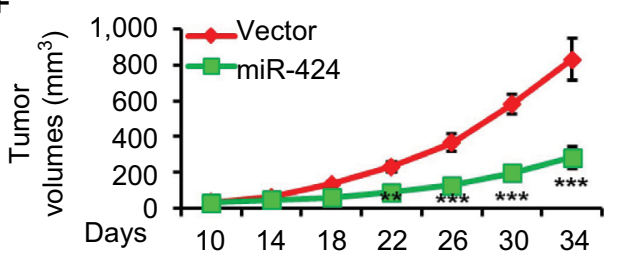

Figure I miR-424 is downregulated in CRC tissues and cell lines and suppressed cancer cell growth in vitro and in vivo.

Notes: (A) miR-424 expression was evaluated using RT-qPCR in total RNAs derived from 53 matched pairs of CRC tissues and corresponding normal colon mucosa. N stands for normal and CA for CRC sample. U6 served as the endogenous control for miR-424. Data are presented as fold change (2- ${ }^{\Delta C T}$ ). (B) RT-qPCR analysis of miR-424 in total RNA from indicated CRC cell lines and the normal colorectal tissues. The data shown are fold changes of individual miRNA expression in each CRC line relative to the normal tissues. (C, D) The effects of miR-424 on CRC cell growth (HCTII6, C; RKO, D) were measured via a CCK-8 assay at the time indicated. The results are presented as the mean \pm SD of the values obtained in three independent experiments. (E, F) Overexpression of miR-424 inhibits tumor formation in nude mice. HCTII 6 cells $\left(5 \times 10^{6}\right)$ stably overexpressing either $\mathrm{p}$-miR-424 or $\mathrm{p}$-scramble control were injected subcutaneously into the flanks of nude mice ( $\mathrm{n}=3$ in each group). The tumor volume was measured every 4 days starting from day 10 until the mice were killed 34 days after transplantation and assessed using the formula $(L \times D \times W) \times \pi / 6$, where $L$ is the length, $D$ is the depth, and $W$ is the width. Photographs show representative features of the tumor growth (E) and line graph shows the mean tumor volume of the injected mice (F). $* P<0.05 ; * * P<0.01 ; * * * P<0.001$.

Abbreviations: $C R C$, colorectal cancer; RT-qPCR, real-time quantitative reverse transcriptase-PCR.

\section{MiR-424 sensitizes CRC cells to apoptosis}

To further explore the mechanism of miR-424 tumor suppressor role, we examined the effect of miR-424 on apoptosis. As expected, we demonstrate that, compared to negative control group, overexpression of miR-424 caused a significant increase (2.7- and 1.8-fold; Figure 2A, B) in early apoptotic and late apoptotic/necrotic activity of both HCT116 and RKO cells, as determined by Annexin V staining. Moreover, the noted effect of miR-424 on apoptosis was confirmed, as increased $\mathrm{p} 53$ expression was induced in HCT116 and RKO cells that overexpressed miR-424 (Figure 2C).

\section{Gene expression analysis for miR-424 according to microarray data}

To explore the effects of miR-424 on CRC growth and apoptosis, we generated a gene expression microarray of cells transfected with miR-424 mimic or miR-NC for 48 hours. An absolute fold change of $>2$ and a $P$-value of $<0.05$ were designated as significantly differentially expressed genes. We finally identified 499 differentially expressed genes upon miR-424 reintroduction, with 241 upregulated and 258 downregulated (Figure $3 \mathrm{~A}$, Table S1). To probe the biologic relevance of the above 499 identified differentially expressed genes obtained from miR-424 vs miR-NC, we then performed IPA. Canonical pathway analysis indicated clear patterns of enriched pathways for miR-424 (Figure 3B). We revealed that PTEN and $\mathrm{p} 53$ signaling pathways were major pathways being activated by miR-424 in CRC cells. Further analysis focused on molecular and function analysis showed that cell survival, growth, and development were mainly influenced (Figure 3C). Moreover, association with gene networks showed that broad differences in genes associated with nuclear factor kappa B (NF-אB), ERK1/2, and ATF4 were observed as the top deregulated networks with miR424 overexpression (Figure 4). Overall, these enrichments underscore the miR-424 signaling-associated pathways. 
Table I Association between miR-424 expression and clinicopathologic parameters of colorectal cancer patients

\begin{tabular}{|c|c|c|c|c|}
\hline \multirow[t]{2}{*}{ Characteristics } & \multirow[t]{2}{*}{$\begin{array}{l}\text { Total } \\
(n=53)\end{array}$} & \multicolumn{2}{|c|}{$\begin{array}{l}\text { miR-424 } \\
\text { expression }\end{array}$} & \multirow[t]{2}{*}{$P$-value } \\
\hline & & Low & High & \\
\hline Gender & & & & 0.901 \\
\hline Male & 29 & 14 & 15 & \\
\hline Female & 24 & 12 & 12 & \\
\hline Age, years & & & & 0.875 \\
\hline$<65$ & 23 & 11 & 12 & \\
\hline$\geq 65$ & 30 & 15 & 15 & \\
\hline Tumor site & & & & 0.206 \\
\hline Colon & 30 & 17 & 13 & \\
\hline Rectum & 23 & 9 & 14 & \\
\hline Histology & & & & 0.704 \\
\hline Adenocarcinoma & 46 & 22 & 24 & \\
\hline Mucinous & 7 & 4 & 3 & \\
\hline Tumor size $(\mathrm{cm})$ & & & & 0.845 \\
\hline$<5$ & 29 & 13 & 16 & \\
\hline$\geq 5$ & 24 & 13 & 11 & \\
\hline Differentiation & & & & 0.066 \\
\hline Well-moderate & 39 & 16 & 23 & \\
\hline Poor, mucinous & 14 & 10 & 4 & \\
\hline Local tumor invasion & & & & 0.332 \\
\hline TI & I & 0 & I & \\
\hline $\mathrm{T} 2$ & 7 & 2 & 5 & \\
\hline T3 & 18 & 8 & 10 & \\
\hline T4 & 27 & 16 & 11 & \\
\hline Lymph node & & & & 0.019 \\
\hline metastasis & & & & \\
\hline Negative & 31 & 11 & 20 & \\
\hline Positive & 22 & 15 & 7 & \\
\hline Distant metastasis & & & & 0.192 \\
\hline Negative & 48 & 22 & 26 & \\
\hline Positive & 5 & 4 & 1 & \\
\hline TNM stage & & & & 0.028 \\
\hline I-II & 26 & 9 & 19 & \\
\hline III & 22 & 13 & 7 & \\
\hline IV & 5 & 4 & I & \\
\hline
\end{tabular}

Note: Bold values indicate statistically significant.

\section{Validation of the microarray data}

To examine the accuracy of these differentially expressed genes identified by microarray, we selected 12 genes from the 499 differentially expressed genes for our validation (Figure 5A). RT-qPCR validation showed that all these 12 genes (all $P<0.05$ ) we assayed were detected in the identical expression trend between miR-424 mimic and miR-NC group in HCT116 cells as determined by microarray (Figure 5B). Moreover, nine of them were also expressed in the similar pattern in RKO cells with miR-424 overexpression (Figure 5C). Among them, AKT3 and PSAT1, two main members of the top networks, exhibited the greatest fold change compared to the control cells. As miR-424 is proapoptotic miRNA in
$\mathrm{CRC}$, the strong change in AKT3 and PSAT1 expression suggests that they have a vital role in CRC.

\section{Identification of AKT3 and PSATI as main target genes of miR-424}

We then examined the expression of AKT3 and PSAT1 mRNA levels in the same CRC patients by RT-qPCR. As shown in Figure 6A, both AKT3 and PSAT1 expression levels were significantly elevated in CRC tissues compared to the normal control tissues. We also analyzed the association of AKT3 and PSAT1 with miR-424 expression and found that they were both negatively correlated with miR-424 expression (Figure 6B). We next sought to identify whether 3'UTR of human AKT3 or PSAT1 contains a putative-binding site for miR-424. As shown in Figure 6C, we found that both AKT3 and PSAT1 have putative miR-424-binding sites in their 3'UTR using two different algorithms TargetScan and microRNA.org.

Overexpression of miR-424 in HCT116 cells greatly changed the protein levels of AKT3 and PSAT1 (Figure 6D). Similar results were also found in RKO cells (Figure 6D). Then, we constructed their wild-type (WT) or mutant 3'UTR region and cotransfected with miR-424 or miR-NC into $293 \mathrm{~T}$ cells. Our results showed that miR-424 clearly reduced the luciferase activity of the WT-AKT3 and PSAT1 reporter, as compared to miR-NC groups (Figure 6E). However, the mutation of the putative miR-424-binding sites in the $3^{\prime}$ UTR region significantly abrogated the repression of the luciferase actively caused by miR-424 overexpression (Figure 6E). These data suggest that miR-424 may inhibit AKT3 and PSAT1 expression through targeting their 3'UTR.

\section{AKT3 and PSATI silencing recapitulates the effects of miR-424 in CRC cells}

As miR-424 regulates CRC cell proliferation/apoptosis and suppresses AKT3 and PSAT1 expression, we further examined whether miR-424 exerted its biologic functions via the two target regulations. To verify this, we used gene-specific siRNAs targeting AKT3 and PSAT1, respectively. CCK8 assay showed that both knockdown of AKT3 and PSAT1 clearly suppressed the proliferation of both HCT116 and RKO cells, respectively (Figure 7A, B). Moreover, inhibition of AKT3 or PSAT1 also increased apoptosis of both HCT116 and RKO cells (Figure 7C, D). The suppression of proliferation and enhancement of apoptosis observed by knockdown of AKT3 and PSAT1 were similar to the phenotype induced by forced expression of miR-424 in CRC cells, suggesting that AKT3 and PSAT1 are functional targets of miR-424 in CRC cells. 
A
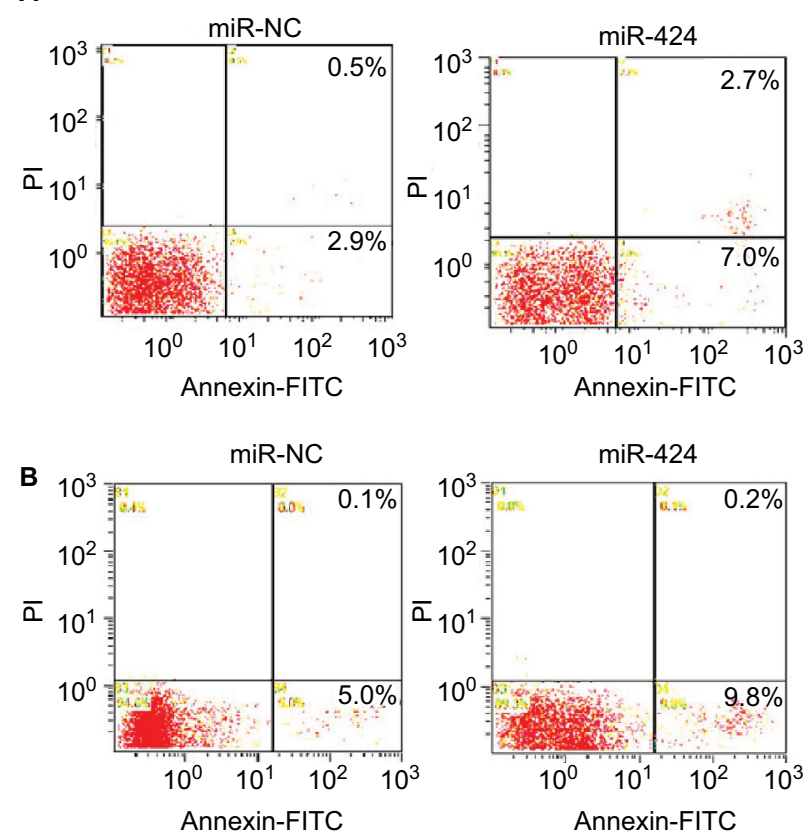

C
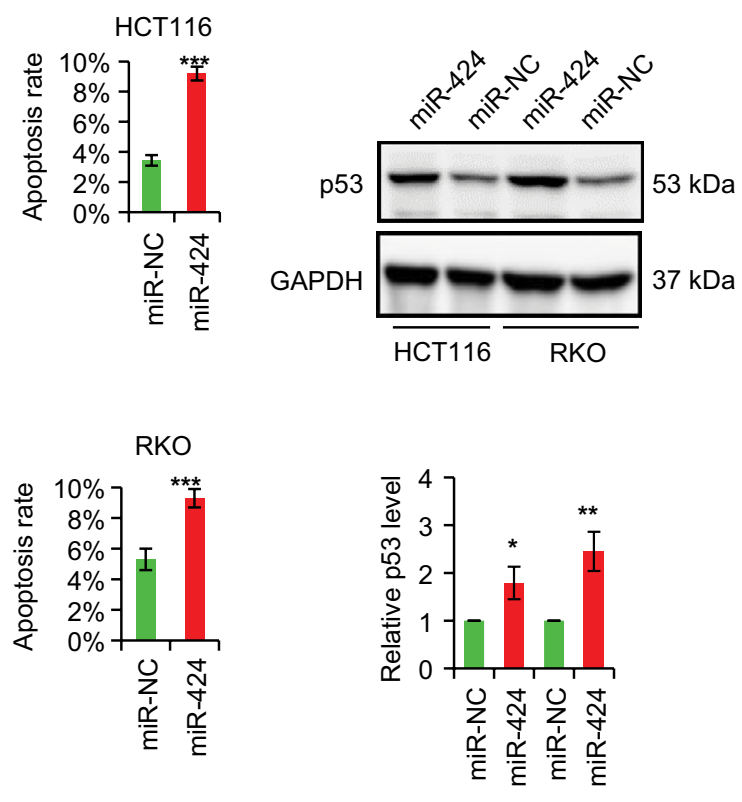

Figure 2 miR-424 induces apoptosis and enhances $\mathrm{p} 53$ expression in CRC cells

Notes: (A, B) Annexin V assay of apoptosis. HCTII6 and RKO cells were transfected with miR-424 mimic or miR-NC for 72 hours and stained with Annexin $V$ and propidium iodide. Both early and late apoptotic cells are combined for analysis. The values are shown as mean \pm SD of three independent experiments. (C) Western blotting analysis for $\mathrm{P} 53$ in miR-424- or miR-NC-transfected HCTII 6 and RKO cells for 72 hours using GAPDH as a loading control. $* P<0.05$; $* * P<0.01$; $* * * P<0.00 \mathrm{I}$.

Abbreviations: FITC, fluorescein isothiocyanate; GAPDH, glyceraldehyde 3-phosphate dehydrogenase; miR-NC, negative control miRNA; PI, propidium iodide.

A

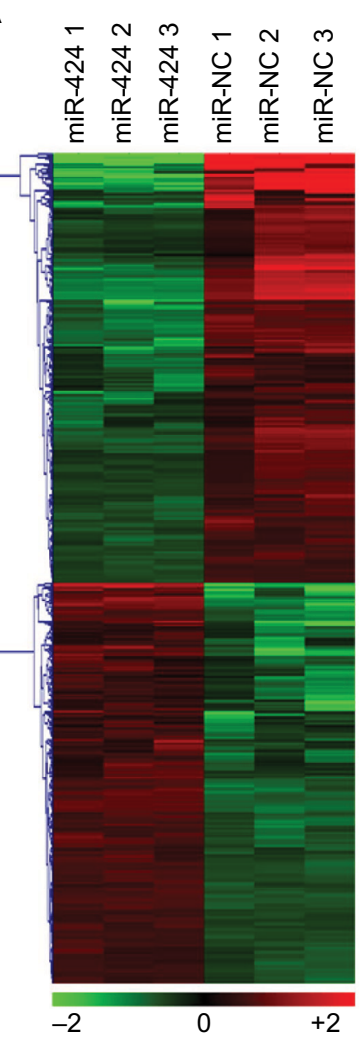

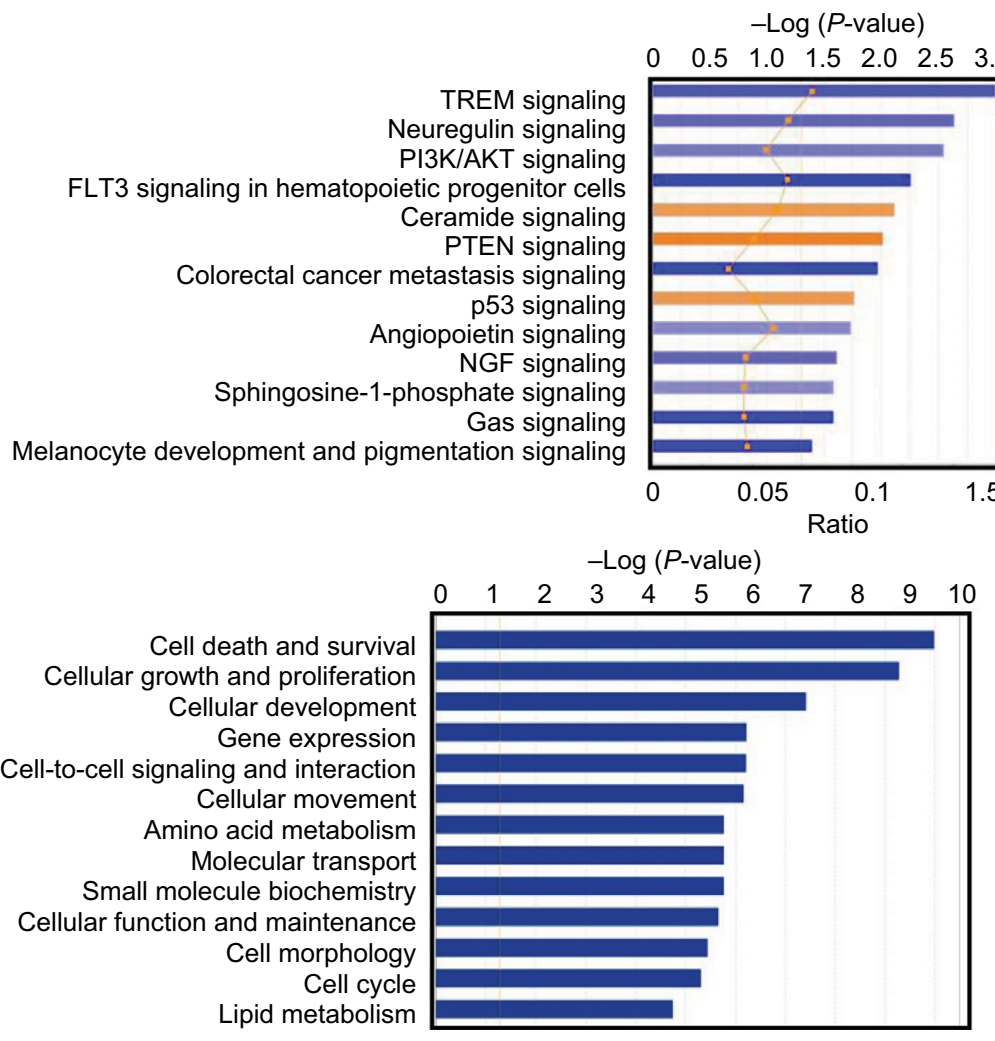

Figure 3 Gene expression profiling of miR-424 and pathway enrichment analysis in HCTII6 cells.

Notes: (A) Heatmaps of hierarchical gene clustering demonstrating miR-424-regulated transcriptome profiles. After overnight culture, HCTII6 cells were transfected with miR-424 and miR-NC for 48 hours, and total RNA was obtained for microarray analysis. For all array analyses, an absolute fold change $\geq 2.0$ and a $P$-value $<0.05$ cutoff were applied. The heatmap depicts the 499 mRNAs that were differentially expressed between miR-424 and miR-NC groups in HCTII6 cells. (B) Canonical pathway analysis of the 499 differential changes using IPA showing 13 most significant activated (orange) and inhibited (blue) pathways in response to miR-424 expression. (C) Molecular and cell function analysis of the identified 499 genes showing 13 main influenced functional pathways upon miR-424 expression.

Abbreviation: IPA, Ingenuity pathway analysis; miR-NC, negative control miRNA. 


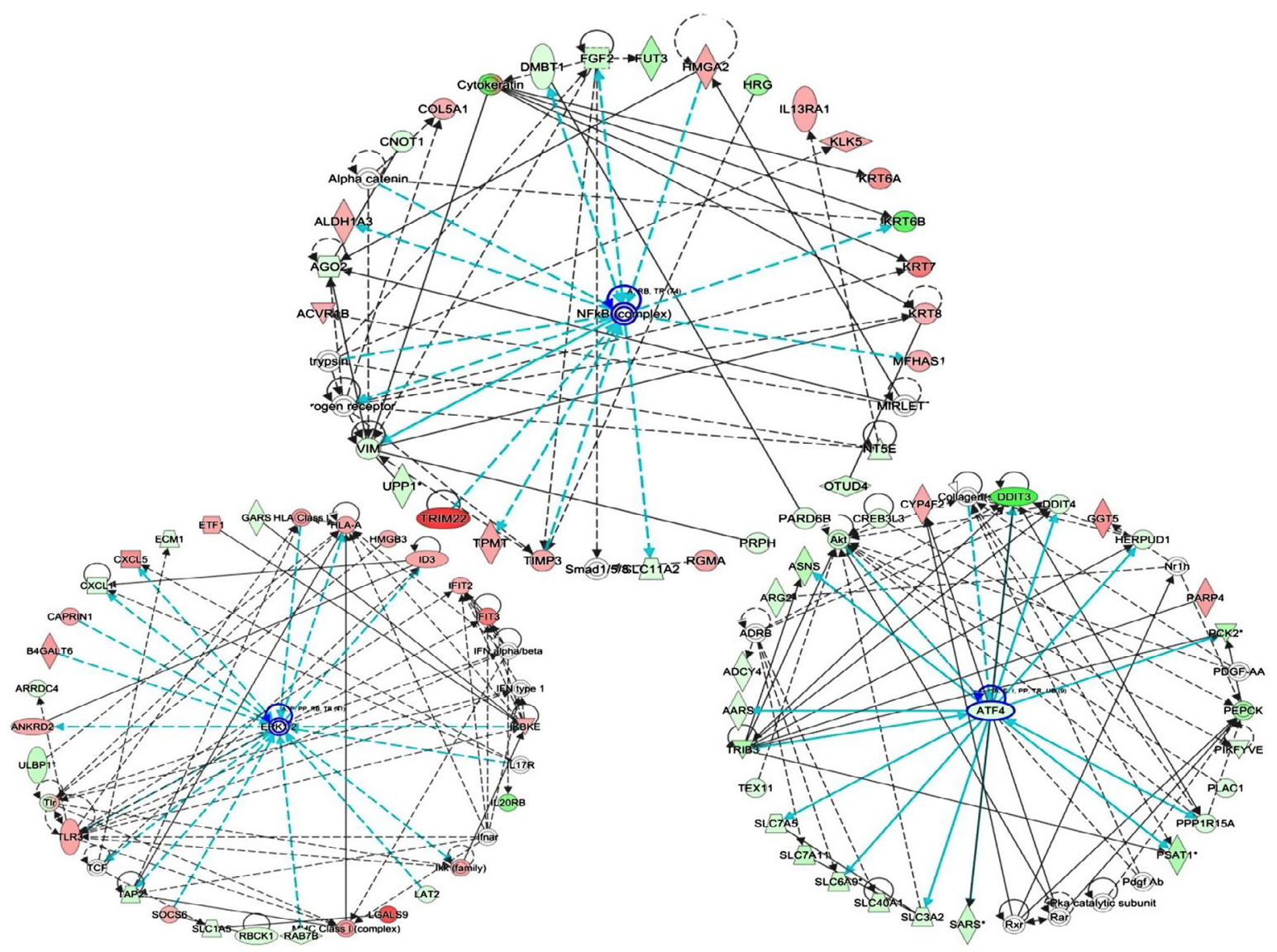

Figure 4 Network analysis of 499 differential changes by miR- 424 in HCTII 6 cells.

Notes: Top three networks of interest (nuclear factor kappa B [NF-KB], ERK, and ATF4) were generated. Green, lower expression upon miR-424 expression; red, higher expression upon miR-424 expression.

A

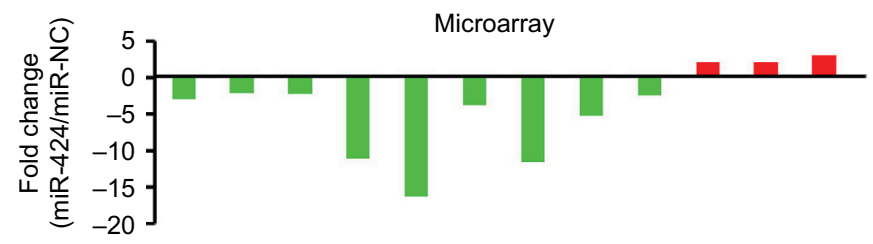

B

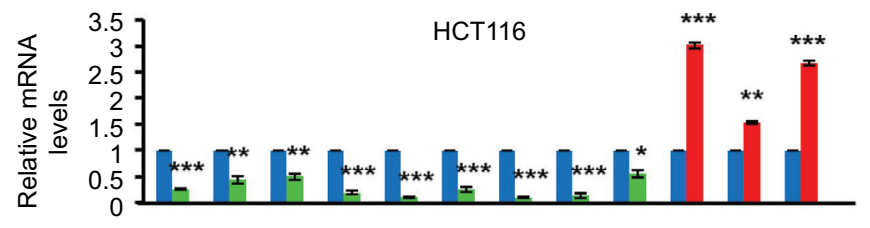

C

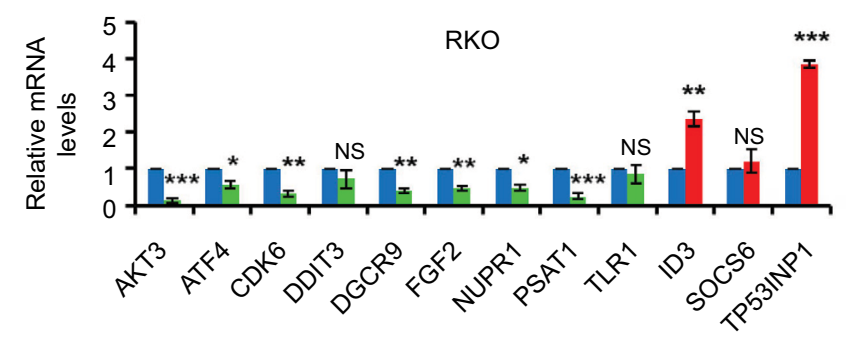

Figure 5 Experimental validation of microarray data in CRC cell lines.

Notes: (A) Distribution of gene expression levels of 12 selected genes from the 499 altered genes, measured by microarray with fold change. (B, C) SYBR RT-qPCR validation of the 12 genes in both HCTII6 (B) and RKO (C) cells with or without miR-424 expression at 48 hours post-transfection. The relative mRNA levels are presented as the fold change compared to miR-NC control group. Data are shown as the mean $\pm \mathrm{SD}$ of three independent experiments. $* P<0.05 ; * * P<0.01 ; * * * P<0.001$.

Abbreviations: CRC, colorectal cancer; miR-NC, negative control miRNA; NS, not significant; RT-qPCR, real-time quantitative reverse transcriptase-PCR. 
A

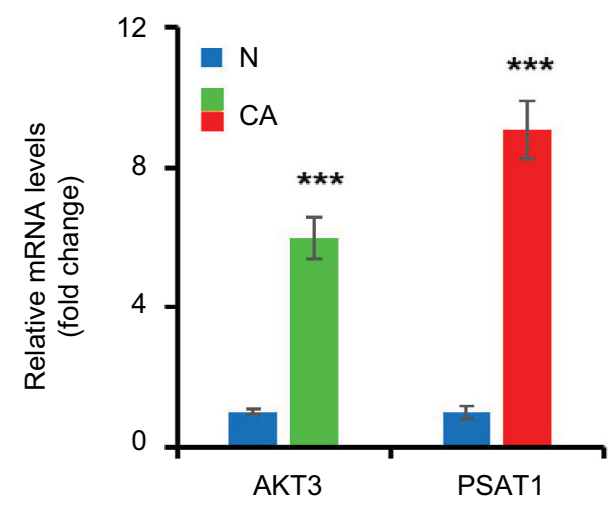

B

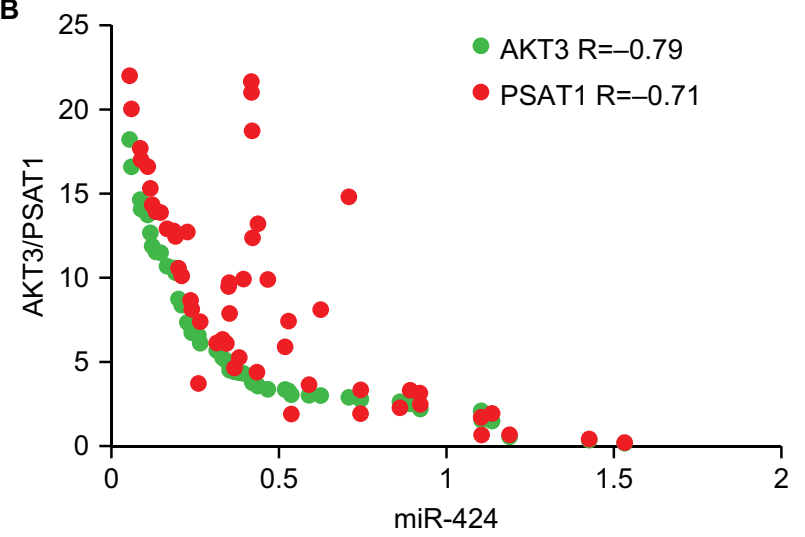

C

\begin{tabular}{|l|c|}
\hline $\begin{array}{l}\text { Position 235-242 of AKT3 3' UTR } \\
\text { hsa-miR-424 }\end{array}$ & $\begin{array}{c}\text { 5'...AAGGUCUCAUGCUGUUGCUGCUA... } \\
\text { I। I I I I । }\end{array}$ \\
\hline
\end{tabular}

\begin{tabular}{l|l} 
Position 264-271 of PSAT1 3' UTR & 5'...CGCGUAUUUUGCCUUUGCUGCUA...
\end{tabular}

III || | |

hsa-miR-424

3' AAGUUUUGUACUUAACGACGAC

D

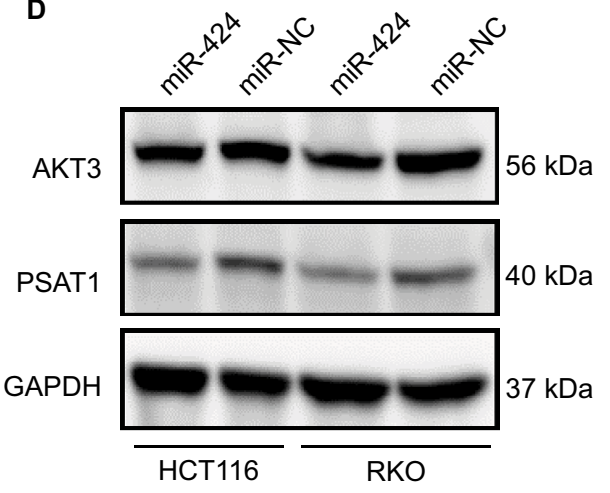

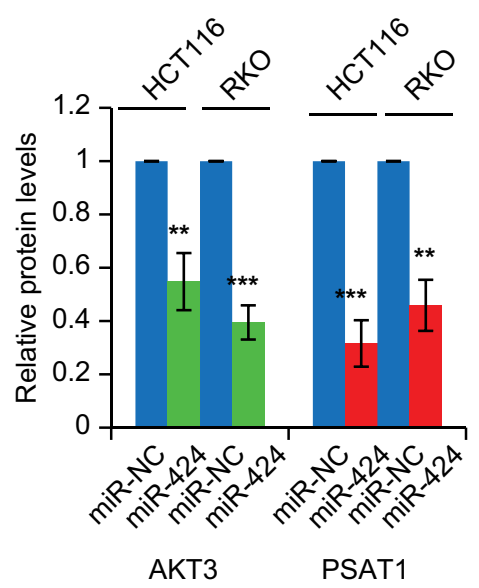

E

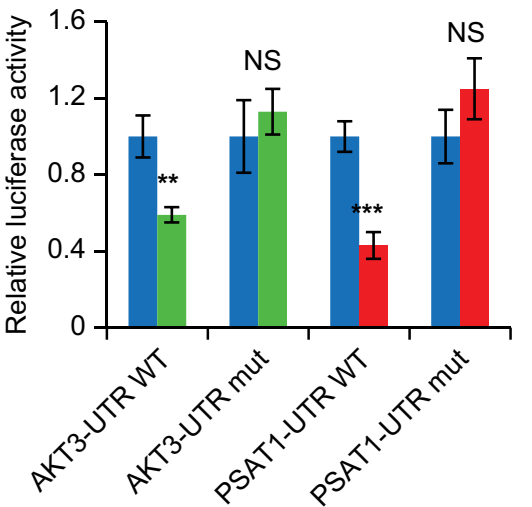

Figure 6 miR-424 targets AKT3 and PSATI in HCTII 6 and RKO cells.

Notes: (A) The expression levels of AKT3 and PSATI mRNA were determined in 53 matched pairs of CRC tissues and corresponding normal colon mucosa by RT-qPCR. $\mathrm{N}$ stands for normal and CA for CRC samples. Data are presented as fold change. (B) Correlation between AKT3 or PSATI and miR-424 expression in CRC patients. (C) The predicted miR-424-binding sites and sequences in the 3'UTR of AKT3 and PSATI mRNAs are shown. (D) Western blotting analysis of AKT3 and PSATI expressions in HCTII6 and RKO cells transfected with miR-424 or miR-NC at 72 hours post-transfection. GAPDH was used as a loading control. (E) Analysis of luciferase activity. PmirGLO-wild-AKT3 3'UTR, pmirGLO-mutant-AKT3 3'UTR, pmirGLO-wild-PSATI 3'UTR, or pmirGLO-mutant-PSATI 3'UTR was cotransfected into 293 T cells with miR-424 or miR-NC. The luciferase activity was determined. Renilla luciferase activity was used as an internal control. Data represent the average of three independent experiments. NS, not significant; $* * P<0.01 ; * * * P<0.001$.

Abbreviations: AKT3, AKT serine/threonine kinase 3; CRC, colorectal cancer; GAPDH, glyceraldehyde 3-phosphate dehydrogenase; miR-NC, negative control miRNA; UTR, untranslated region.

\section{Discussion}

In recent years, miRNAs have been found to control cell proliferation in CRC development and progression. In this report, we presented evidence that miR-424 was frequently reduced in both CRC tissues and cell lines. Restoration of miR-424 significantly inhibited CRC cell growth in vitro and in vivo, and increased apoptosis. Moreover, gain of function of miR-424, followed by gene expression microarray, 
A

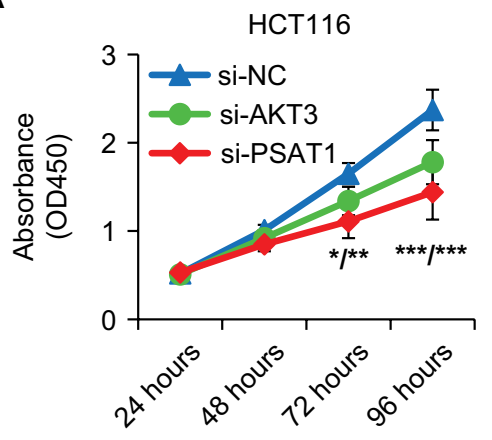

B

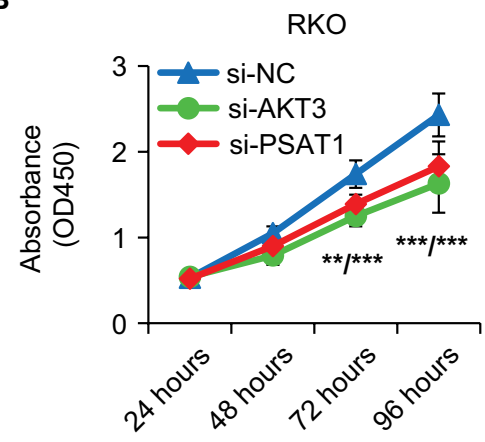

C

HCT116
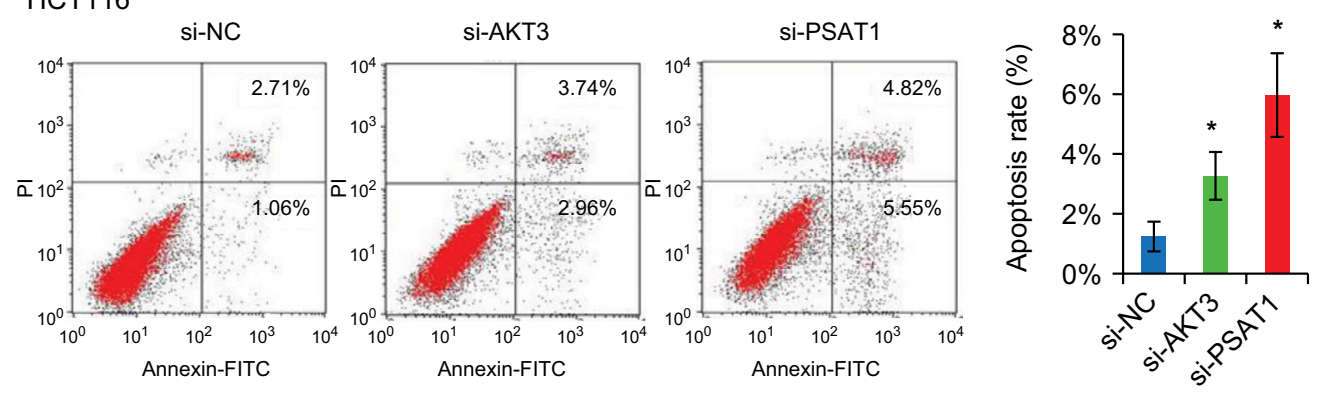

D
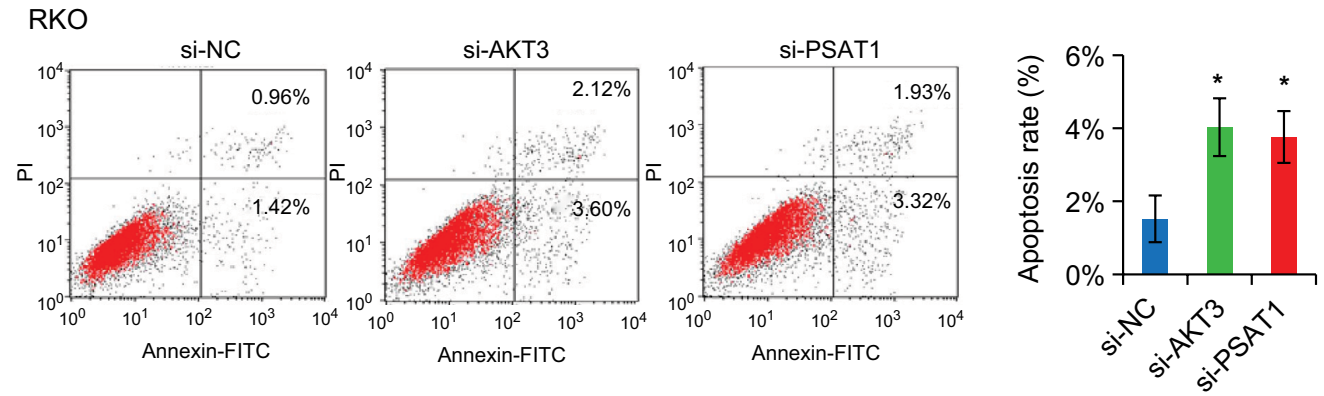

Figure 7 Knockdown of AKT3 and PSATI recapitulates the effects of miR-424 in CRC cells.

Notes: (A, B) Specific siRNA knockdown of AKT3 or PSATI in HCTII6 (A) and RKO (B) cells was evaluated by CCK-8 assays at the time indicated. The results are presented as the mean \pm SD of the values obtained in three independent experiments. (C, D) Apoptosis of HCTII 6 and RKO cells was noticeably accelerated following transfection with si-AKT3 or si-PSATI, compared to si-NS control. The values are shown as mean \pm SD of three independent experiments. $* P<0.05$; $* * P<0.01$; $* * * P<0.00$ I. Abbreviations: AKT3, AKT serine/threonine kinase 3; CRC, colorectal cancer; FITC, fluorescein isothiocyanate; NS, not significant; si-AKT3, AKT3-specific siRNA; Si-NS, nonspecific siRNA; si-PSATI, PSATI-specific siRNA.

identified a set of downstream targets of miR-424. We also showed major pathways activated or repressed by miR-424. Among them, we finally determined AKT3 and PSAT1 as two functional targets of miR-424 in CRC cells. Our results provide a more comprehensive understanding of the tumor suppressor role of miR-424 in CRC and highlight the possibility of miR-424 as a novel anticancer target.

MiR-424 is a member of the miR-15/107 family and has pleiotropic roles in cell division, metabolism, angiogenesis, and stress response in vertebrate species. ${ }^{12-17}$ It also has important roles in many cancer types. Studies have shown that miR-424 often acts as a tumor suppressor miRNA, and its expression is aberrantly downregulated in cervical cancer, breast cancer, renal cancer, and hepatocellular carcinoma. ${ }^{18-21}$ However, the effect of miR-424 on tumorigenesis is still controversial, as some studies revealed that miR-424 suppressed cell proliferation, induced apoptosis, caused cell cycle arrest, or suppressed invasion, ${ }^{18,19,21}$ whereas others reported the promoting role of miR-424 in cancer cell survival, ${ }^{22}$ suggesting that miR-424 might have an environment-dependent effect. Here, we provide new evidence that miR-424 is tumor suppressive in CRC and more importantly reveal the functional role of miR-424 on cancer cell growth and apoptosis. Our study may expand our understanding of the role of miR-424 in cancer development and support the rationale of targeting miR-424 for cancer treatment. 
A single miRNA often regulates many gene targets. ${ }^{23-25}$ Microarray can detect potential miRNA:mRNA interactions, and using microarrays to screen for alterations in gene expression following transfection of miR-424 mimics would show the physiologic miR-424 functions. Our gene expression analysis by microarray showed that a set of 258 annotated genes were significantly downregulated by miR424 in HCT116 cells. Validation of these targets further confirmed the microarray results. Furthermore, IPA analysis showed that miR-424 mainly modulated NF-KB, ERK1/2, and ATF4 networks. Importantly, we identified AKT3 and PSAT1 expression, which is under ATF4 transcriptional control, to be abrogated upon miR-424 expression.

The serine/threonine protein kinase AKT regulates cell growth, survival, proliferation, protection from apoptosis, control of DNA damage responses, metabolism, migration, and metastasis, all of which are hallmarks of cancer. ${ }^{26}$ There are three members of the AKT family, that is, AKT1-3. ${ }^{26}$ Interestingly, although all of the AKTs share a considerable homology, they may play different, or even opposite, roles in tumorigenesis. Among the AKT family, AKT1 has been shown to be overexpressed in breast cancer, ${ }^{27} \mathrm{AKT} 2$ in CRC, ${ }^{28}$ and AKT 3 has been found to be overexpressed in melanoma. ${ }^{29}$ In our study, we revealed a tumor-promoting role of AKT3 in $\mathrm{CRC}$ and identified it as a direct target of miR-424. In consistent with our finding, Yang et al also found that miR-424 could target AKT3 in hepatocellular carcinoma. ${ }^{12}$ We further demonstrated that miR-424 also targeted PSAT1. Importantly, targeting PSAT1 reduced cell proliferation and induced apoptosis in both HCT116 and RKO cells, suggesting miR424 as a key player of CRC development. PSAT1 has been recently found to be overexpressed in many cancers, such as breast cancer and CRC. ${ }^{30}$ We provide an miRNA-mediated mechanistic explanation for PSAT1 overexpression in CRC, as it acts as a functional miR-424 target.

\section{Conclusion}

We provide a new understanding of the roles of miR-424 in CRC. In particular, we identified miR-424 as crucial regulators of CRC cell proliferation and apoptosis through combinatorial targeting of multiple oncogenic genes, involving AKT3 and PSAT1. The miR-424-based therapy might be an important treatment for CRC and our results may have important translational implications.

\section{Acknowledgment}

This study was funded by grants from National Natural Science Foundation of China (81702552) and Medical and
Health Science and Technology Project of Zhejiang Province (2017KY099).

\section{Disclosure}

The authors report no conflicts of interest in this work.

\section{References}

1. Torre LA, Bray F, Siegel RL, Ferlay J, Lortet-Tieulent J, Jemal A. Global cancer statistics, 2012. CA Cancer J Clin. 2015;65(2):87-108.

2. Center MM, Jemal A, Smith RA, Ward E. Worldwide variations in colorectal cancer. CA Cancer J Clin. 2009;59(6):366-378.

3. Desantis CE, Lin CC, Mariotto AB, et al. Cancer treatment and survivorship statistics, 2014. CA Cancer J Clin. 2014;64(4):252-271.

4. Bartel DP. MicroRNAs: target recognition and regulatory functions. Cell. 2009;136(2):215-233.

5. Iorio MV, Croce CM. MicroRNA involvement in human cancer. Carcinogenesis. 2012;33(6):1126-1133.

6. Pasquinelli AE. MicroRNAs and their targets: recognition, regulation and an emerging reciprocal relationship. Nat Rev Genet. 2012;13(4): 271-282.

7. Lin S, Gregory RI. MicroRNA biogenesis pathways in cancer. Nat Rev Cancer. 2015;15(6):321-333.

8. Sayed D, Abdellatif M. MicroRNAs in development and disease. Physiol Rev. 2011;91(3):827-887.

9. Xu J, Fang Y, Wang X, et al. CUL2 overexpression driven by CUL2/ E2F1/miR-424 regulatory loop promotes HPV16 E7 induced cervical carcinogenesis. Oncotarget. 2016;7(21):31520-31533.

10. Fang Y, Yu H, Liang X, Xu J, Cai X. Chk1-induced CCNB1 overexpression promotes cell proliferation and tumor growth in human colorectal cancer. Cancer Biol Ther. 2014;15(9):1268-1279.

11. Xu J, Wan X, Chen X, et al. miR-2861 acts as a tumor suppressor via targeting EGFR/AKT2/CCND1 pathway in cervical cancer induced by human papillomavirus virus 16 E6. Sci Rep. 2016;6:28968.

12. Yang H, Zheng W, Shuai X, et al. MicroRNA-424 inhibits Akt3/E2F3 axis and tumor growth in hepatocellular carcinoma. Oncotarget. 2015;6(29):27736-27750.

13. Finnerty JR, Wang WX, Hébert SS, Wilfred BR, Mao G, Nelson PT. The miR-15/107 group of microRNA genes: evolutionary biology, cellular functions, and roles in human diseases. $\mathrm{J} \mathrm{Mol} \mathrm{Biol.}$ 2010;402(3):491-509.

14. Lee A, Papangeli I, ParkY, et al. A PPAR $\gamma$-dependent miR-424/503-CD40 axis regulates inflammation mediated angiogenesis. Sci Rep. 2017;7(1): 2528.

15. Zhang D, Shi Z, Li M, Mi J. Hypoxia-induced miR-424 decreases tumor sensitivity to chemotherapy by inhibiting apoptosis. Cell Death Dis. 2014;5:e1301.

16. Peng HY, Jiang SS, Hsiao JR, et al. IL-8 induces miR-424-5p expression and modulates SOCS2/STAT5 signaling pathway in oral squamous cell carcinoma. Mol Oncol. 2016;10(6):895-909.

17. Drasin DJ, Guarnieri AL, Neelakantan D, et al. TWIST1-induced miR424 reversibly drives mesenchymal programming while inhibiting tumor initiation. Cancer Res. 2015;75(9):1908-1921.

18. Xu J, Li Y, Wang F, et al. Suppressed miR-424 expression via upregulation of target gene Chk1 contributes to the progression of cervical cancer. Oncogene. 2013;32(8):976-987.

19. Rodriguez-Barrueco R, Nekritz EA, Bertucci F, et al. miR-424(322)/503 is a breast cancer tumor suppressor whose loss promotes resistance to chemotherapy. Genes Dev. 2017;31(6):553-566.

20. Chen B, Duan L, Yin G, Tan J, Jiang X. Simultaneously expressed miR424 and miR-381 synergistically suppress the proliferation and survival of renal cancer cells-Cdc2 activity is up-regulated by targeting WEE1. Clinics (Sao Paulo). 2013;68(6):825-833.

21. Yu L, Ding GF, He C, Sun L, Jiang Y, Zhu L. MicroRNA-424 is downregulated in hepatocellular carcinoma and suppresses cell migration and invasion through c-Myb. PLoS One. 2014;9(3):e91661. 
22. Wei S, Li Q, Li Z, Wang L, Zhang L, Xu Z. miR-424-5p promotes proliferation of gastric cancer by targeting Smad3 through TGF- $\beta$ signaling pathway. Oncotarget. 2016;7(46):75185-75196.

23. Lim LP, Lau NC, Garrett-Engele P, et al. Microarray analysis shows that some microRNAs downregulate large numbers of target mRNAs. Nature. 2005;433(7027):769-773.

24. Lewis BP, Shih IH, Jones-Rhoades MW, Bartel DP, Burge CB. Prediction of mammalian microRNA targets. Cell. 2003;115(7): 787-798.

25. Bartel DP, Chen CZ. Micromanagers of gene expression: the potentially widespread influence of metazoan microRNAs. Nat Rev Genet. 2004;5(5):396-400.

26. Gonzalez E, McGraw TE. The Akt kinases: isoform specificity in metabolism and cancer. Cell Cycle. 2009;8(16):2502-2508.
27. Stål $\mathrm{O}$, Pérez-Tenorio G, Akerberg L, et al. Akt kinases in breast cancer and the results of adjuvant therapy. Breast Cancer Res. 2003;5(2):R37-44.

28. Roy HK, Olusola BF, Clemens DL, et al. AKT proto-oncogene overexpression is an early event during sporadic colon carcinogenesis. Carcinogenesis. 2002;23(1):201-205.

29. Stahl JM, Sharma A, Cheung M, et al. Deregulated Akt3 activity promotes development of malignant melanoma. Cancer Res. 2004;64(19): 7002-7010.

30. Vié N, Copois V, Bascoul-Mollevi C, et al. Overexpression of phosphoserine aminotransferase PSAT1 stimulates cell growth and increases chemoresistance of colon cancer cells. Mol Cancer. 2008;7:14.
Cancer Management and Research

\section{Publish your work in this journal}

Cancer Management and Research is an international, peer-reviewed open access journal focusing on cancer research and the optimal use of preventative and integrated treatment interventions to achieve improved outcomes, enhanced survival and quality of life for the cancer patient The manuscript management system is completely online and includes

\section{Dovepress}

a very quick and fair peer-review system, which is all easy to use. Visit http://www.dovepress.com/testimonials.php to read real quotes from published authors. 